\title{
THE CAUCHY PROBLEM FOR THE SYSTEM OF \\ ELASTICITY
}

@ F.M. Bolikulov, I.E.Niyozov

bolikulovfurkat@mail.ru,iqboln@mail.ru

\section{УДК 517.946}

DOI: 10.33184/mnkuomsh1t-2021-10-06.70.

В данной работе рассматривается задача аналитического продолжения решения системы уравнений моментной теории упругости в пространственной области через ее значения и значения ее напряжений на части границы этой область, т. е. проблема Коши.

Ключевъе слова: Задача Коши, системная теория упругости, эллиптическая система, некорректная задача, Карлеман матрица, регуляризация.

In this paper, we consider the problem of the analytical continuation of the solution of the system of equations of the moment theory of elasticity in the spatial domain in terms of its values and the values of its stresses on a part of the boundary of this domain, i.e. the Cauchy problem.

Keywords: Cauchy problem, system theory of elasticity, elliptic system, ill-posed problem, Carleman matrix, regularization.

In this paper, we considered the problem of analytical continuation of the solution of the system equations of the moment theory of elasticity in spacious bounded domain from its values and values of its strains on part of the boundary of this domain, i.e., the Cauchy's problem.

System equation of moment theory elasticity is elliptic. Therefore the problem Cauchy for this system is ill-posed. For ill-posed problems, one does not prove the existence theorem: the existence is assumed a priori. Moreover, the solution is assumed to belong to some given subset of the function space, usually a compact one [1]. The uniqueness of the solution follows from the general Holmgren theorem. On establishing uniqueness in the article studio of ill-posed problems, one comes across important questions concerning the derivation of estimates of conditional stability and the construction of regularizing operators.

Bolikulov Furkat Mamarajabovich, magistr, Samarkand State University (Samarkand, Uzbekistan); Фуркат Боликулов (Самаркандский госуниверситет, Самарканд, Узбекистан)

Niyozov Ikbol Ergashevich, assistant professor, Samarkand State University (Samarkand, Uzbekistan); Икбол Ниёзов (Самаркандский госуниверситет, Самарканд, Узбекистан) 
Our aim is to construct an approximate solution using the Carleman function method [2].

Let $x=\left(x_{1}, \ldots, x_{m}\right)$ and $y=\left(y_{1}, \ldots, y_{m}\right)$ be points of the Euclidean space $E^{m}, D$ a bounded simply connected domain in $E^{m}$, with piecewise-smooth boundary consisting of a piece $\Sigma$ of the plane $y_{m}=0$ and a smooth surface S lying in the half-space $y_{m}>0$.

Suppose that vector function $U(x)=\left(u_{1}(x), \ldots, u_{m}(x), v_{1}(x), \ldots, v_{m}(x)\right)=$ $(u(x), v(x))$ satisfied in $D$ the system equations moments theory elasticity:

$$
\left\{\begin{array}{l}
(\mu+\alpha) \Delta u+(\lambda+\mu-\alpha) \text { graddivu }+2 \alpha \text { rot } v+\rho \omega^{2} u=0, \\
(\nu+\beta) \Delta v+(\varepsilon+\nu-\beta) \text { graddiv }+2 \alpha \text { rotu }-4 \alpha v+\theta \omega^{2} v=0,
\end{array}\right.
$$

where $\lambda, \mu, \nu, \beta, \varepsilon, \alpha$ is coefficients which characterizing medium, satisfying the conditions $\mu>0,3 \lambda+2 \mu>0, \alpha>0, \varepsilon>0,3 \varepsilon+2 \nu>0, \beta>0, \theta>$ $0, \rho>0, \omega \in R^{1}$.

Then system (1) maybe write in matrix from in the following way:

$$
M\left(\partial_{x}\right) U(x)=0
$$

A solution $U$ of system (1) in the domain $D$ is said to be regular if $U \in$ $C^{1}(\bar{D}) \bigcap C^{2}(D)$.

Statement of the problem. Find a regular solution $U$ of system (1) in the domain $D$ using its Cauchy data on the surface $S$ :

$$
U(y)=f(y), \quad T\left(\partial_{y}, n(y)\right) U(y)=g(y), \quad y \in S,
$$

where $T\left(\partial_{y}, n(y)\right)$ is the stress operator, $n(y)=\left(n_{1}(y), \ldots, n_{m}(y)\right)$ is the unit outward normal vector on $\partial D$ at a point $y, f=\left(f_{1}, \ldots, f_{2 m}\right), g=$ $\left(g_{1}, \ldots, g_{2 m}\right)$ are given continuous vector functions on $S$.

Suppose that, instead of $f(y)$ and $g(y)$, we are given their approximations $f_{\delta}(y)$ and $g_{\delta}(y)$ with accuracy $\delta, 0<\delta<1$ (in the metric of $C$ ) which do not necessarily belong to the class of solutions. In this paper, we construct a family of functions $U\left(x, f_{\delta}, g_{\delta}\right)=U_{\sigma \delta}(x)$ depending on the parameter $\sigma$ and prove that under certain conditions and a special choice of the parameter $\sigma(\delta)$ the family $U_{\sigma \delta}(x)$ converges in the usual sense to the solution $U(x)$ of problem (1),(3), as $\delta \rightarrow 0$.

The following formula holds [3]:

$$
U(x)=\int_{\partial D}\left(\Pi(y, x, \sigma)\left\{T\left(\partial_{y}, n\right) U(y)\right\}-\left\{T\left(\partial_{y}, n\right) \Pi(y, x, \sigma)\right\}^{*} U(y)\right) d s_{y}, x \in D,
$$

where symbol $*$ is denote of operation transposition, $\Pi(y, x, \sigma)$ Carleman matrix of problem $(1),(3)$ depending on the two points $y, x$ and positive numerical number parameter $\sigma$. 
Now we write out a result that allows us to calculate $U(x)$ approximately if, instead of $U(y)$ and $T\left(\partial_{y}, n\right) U(y)$, their continuous approximations $f_{\delta}(y)$ and $g_{\delta}(y)$ are given on the surface $S$ :

$$
\max _{S}\left|f(y)-f_{\delta}(y)\right|+\max _{S}\left|T\left(\partial_{y}, n\right) U(y)-g_{\delta}(y)\right| \leq \delta, \quad 0<\delta<1 .
$$

We define a function $U_{\sigma \delta}(x)$ by setting

$$
U_{\sigma \delta}(x)=\int_{S}\left[\Pi(y, x, \sigma) g_{\delta}(y)-\left\{T\left(\partial_{y}, n\right) \Pi(y, x, \sigma)\right\}^{*} f_{\delta}(y)\right] d s_{y},
$$

where

$$
\sigma=\frac{1}{x_{m}^{0}} \ln \frac{M}{\delta}, \quad x_{m}^{0}=\max _{D} x_{m}, \quad x_{m}>0 .
$$

Theorem. Let $U(x)$ be a regular solution of system (1) in D satisfying condition

$$
|U(y)|+|T(\partial y, n) U(y)| \leq M, \quad y \in \partial D .
$$

Then the following estimate is valid:

$$
\left|U(x)-U_{\sigma \delta}(x)\right| \leq C(x) \delta^{\frac{x_{m}}{x_{m}^{0}}}\left(\ln \frac{M}{\delta}\right)^{m}, \quad x \in D .
$$

\section{Литература}

1. M.M.Lavrent'ev. Some Ill-Posed Problems of Mathematical Physics [in Russian], Computer Center of the Siberian Division of the Russian Academy of Sciences, Novosibirck (1962) 92p.

2. Sh. Ya.Yarmukhamedov. Cauchy problem for the Laplace equation. Dokl.Acad.Nauk SSSR [Soviet Math.Dokl.],235, No.2,p.281-283.(1977).

3. V.D.Kupradze, T.V.Burchuladze, T.G.Gegeliya, ot.ab. Three-Dimensional Problems of the Mathematical Theory of Elasticity and ... [in Russian], Nauka, Moscow,1976. 